\title{
Non-Darcian Behavior of Pyrolysis Gas in a Thermal Protection System
}

\author{
Alexandre Martin* and Iain D. Boyd亡 \\ University of Michigan, Ann Arbor, Michigan 48109
}

DOI: $10.2514 / 1.44103$

\begin{abstract}
To improve heat and ablation rate modeling of the thermal protection system for reentry vehicles, a material response model with surface ablation and pyrolysis is developed. To accurately model the effects of the pyrolysis gas within the ablator, Darcy's law is replaced by Forchheimer's law for flow through porous media. The use of Forchheimer's law accounts for the inertial effects of the gas and removes any dependency on microscopic parameters, such as pore size. To characterize the flow, the Forchheimer number is proposed because it depends only on macroscopic quantities. To verify and validate the model, comparisons to experimental data and to prior computational results are presented. Applying Ergun's equation to evaluate the inertial parameter of the Forchheimer number, a simple test case is run. For the case of a generic carbon-phenolic ablator subjected to a typical reentry trajectory, conditions for non-Darcian behavior are investigated by way of a parametric study. Finally, the necessary conditions required for gas kinetic energy to be relevant are highlighted.
\end{abstract}

\section{Nomenclature}

$\begin{array}{ll}A & =\text { area } \\ b & =\text { permeability slip parameter } \\ D & =\text { characteristic length of porous media } \\ E & =\text { total energy } \\ F o & =\text { Forchheimer number } \\ h & =\text { enthalpy } \\ K & =\text { permeability } \\ K_{0} & =\text { continuum flow permeability } \\ \dot{m} & =\text { mass flux } \\ \dot{m}^{\prime \prime \prime} & =\text { volumetric mass source term } \\ p & =\text { pressure } \\ q & =\text { surface heat transfer rate } \\ \dot{q}^{\prime \prime} & =\text { internal heat flux } \\ R & =\text { specific gas constant } \\ R e & =\text { Reynolds number } \\ \dot{s} & =\text { recession velocity } \\ T & =\text { temperature } \\ T_{v} & =\text { vibrational temperature } \\ t & =\text { time } \\ v & =\text { velocity } \\ v^{\prime} & =\text { superficial velocity; } v^{\prime}=\phi v \\ V & =\text { volume } \\ x & =\text { coordinates } \\ \beta & =\text { Forchheimer coefficient } \\ \beta_{K} & =\text { Ergun's inertial parameter } \\ \mu & =\text { dynamic viscosity } \\ \rho & =\text { density } \\ \phi & =\text { porosity } \\ & \end{array}$

Subscripts

$c=$ char

$\mathrm{cv}=$ control volume

Presented as Paper 3805 at the 40th Thermophysics Conference, Seattle, WA, 23-26 June 2008; received 2 March 2009; revision received 18 May 2009; accepted for publication 20 May 2009. Copyright $@ 2009$ by Alexandre Martin and Iain D. Boyd. Published by the American Institute of Aeronautics and Astronautics, Inc., with permission. Copies of this paper may be made for personal or internal use, on condition that the copier pay the $\$ 10.00$ per-copy fee to the Copyright Clearance Center, Inc., 222 Rosewood Drive, Danvers, MA 01923; include the code 0887-8722/10 and \$10.00 in correspondence with the CCC.

*Research Fellow, Department of Aerospace Engineering. Member AIAA.

${ }^{\dagger}$ Professor, Department of Aerospace Engineering. Associate Fellow AIAA.

$\begin{array}{ll}\mathrm{cs} & =\text { control surface } \\ g & =\text { gas } \\ \mathrm{kin} & =\text { kinetic energy } \\ s & =\text { solid } \\ \mathrm{st} & =\text { stagnation } \\ v & =\text { virgin } \\ x & =\text { coordinates } \\ \infty & =\text { freestream }\end{array}$

\section{Introduction}

T HE thermal protection system (TPS) of a reentry vehicle is one of the key components of its design. The material used for the TPS can be classified into two main categories: ablative materials, as in the one used on the Apollo missions, and nonablative materials, such as the ceramic tiles used on the space shuttle. The former can also be divided into two subcategories: charring (also know as pyrolyzing) and noncharring ablators. The theory behind the use of ablators is quite simple: the energy absorbed by the removal of material from the surface is not used to heat the TPS, thus keeping the vehicle at a reasonably "cold" temperature. In the case of charring ablators, the main ablative material is a resin which fills the pores of a carbon matrix. Although the matrix may ablate, it usually does not, thus preserving the original geometry of the aerodynamic surface during reentry.

To correctly model the pyrolysis process, the gas generated from resin vaporization must be properly taken into account. Because this gas is expelled into the outer flow boundary layer, its effects are important in many aspects of reentry modeling, such as surface ablation, blowing effects in the boundary layer, chemical reactions with the surface, heat load, etc. The pressure of the gas within the ablator is also an important factor to take into account; if the pressure is too high, chunks of the ablator may "explode" without absorbing the expected amount of heat. This process, called spallation (or delamination if caused by crack formation), may cause unwanted surface modifications and faster surface ablation than anticipated, and thus may lead to the failure of the TPS.

In an attempt to improve the modeling of heat transfer and ablation on hypersonic vehicles, such as reentry capsules, the present study outlines different aspects of the importance of pyrolysis gas modeling. To do so, a thermal response code, named MOPAR (modeling of pyrolysis and ablation response), is developed. First the governing equations describing the phenomena are presented; using realistic reentry conditions, the model is compared to published results from other thermal response codes and is assessed against experimental data. Using a reentry trajectory test case, the contribution of the 
kinetic energy is outlined, and conditions where this contribution may be relevant are discussed. For the same test case, evidence of a non-Darcian flow regime is shown, and the use of the Forchheimer number to categorize the flow type is proposed.

\section{MOPAR: A Material Response Code}

\section{A. Governing Equations and Numerical Scheme}

The material response model implementation presented here is developed using the same methodology as the code created at Sandia National Laboratories [1-4]. The code uses the control volume finite element method to model surface ablation with wall recession, as well as inner decomposition and pyrolysis gas behavior. The model is described by the following four governing equations:

Mixture energy equation:

$$
\frac{\mathrm{d}}{\mathrm{d} t} \int_{\mathrm{cv}} \rho E \mathrm{~d} V+\int_{\mathrm{cs}} \phi \rho_{g} h_{g} v_{g} \mathrm{~d} A+\int_{\mathrm{cs}} \dot{q}^{\prime \prime} \mathrm{d} A-\int_{\mathrm{cs}} \rho h v_{\mathrm{cs}} \mathrm{d} A=0
$$

Solid phase equation:

$$
\frac{\mathrm{d}}{\mathrm{d} t} \int_{\mathrm{cv}} \rho_{s} \mathrm{~d} V-\int_{\mathrm{cs}} \rho_{s} v_{\mathrm{cs}} \mathrm{d} A-\int_{\mathrm{cv}} \dot{m}_{s}^{\prime \prime \prime} \mathrm{d} V=0
$$

Gas-phase continuity equation:

$$
\frac{\mathrm{d}}{\mathrm{d} t} \int_{\mathrm{cv}} \phi \rho_{g} \mathrm{~d} V+\int_{\mathrm{cs}} \phi \rho_{g} v_{g} \mathrm{~d} A-\int_{\mathrm{cs}} \phi \rho_{g} v_{\mathrm{cs}} \mathrm{d} A-\int_{\mathrm{cv}} \dot{m}_{g}^{\prime \prime \prime} \mathrm{d} V=0
$$

Momentum equation:

$$
\begin{aligned}
& \frac{\mathrm{d}}{\mathrm{d} t} \int_{\mathrm{cv}} \phi v_{g} \rho_{g} \mathrm{~d} V+\int_{\mathrm{cs}}\left(\phi \rho_{g} v_{g}^{2}+p\right) \mathrm{d} A-\int_{\mathrm{cs}} \phi v_{g} \rho_{g} v_{\mathrm{cs}} \mathrm{d} A \\
& -\int_{\mathrm{cs}} \mu \frac{\partial v_{g}}{\partial x} \mathrm{~d} A=0
\end{aligned}
$$

The first two equations are solved implicitly on an arbitrary contracting grid using Landau coordinates. Newton's method for nonlinear systems is used to solve both equations sequentially. The third equation is straightforward and does not need to be solved numerically. The final equation is averaged to Darcy's law:

$$
\frac{\partial p}{\partial x}=-\frac{\mu}{K} v_{g}^{\prime}
$$

or Forchheimer's law:

$$
\frac{\partial p}{\partial x}=-\frac{\mu}{K} v_{g}^{\prime}-\beta \rho v_{g}^{\prime 2}
$$

These two equations describe the fluid flow through a porous medium; they both can be explicitly solved for $v_{g}$ and directly integrated in the gas-phase continuity equation. The complete

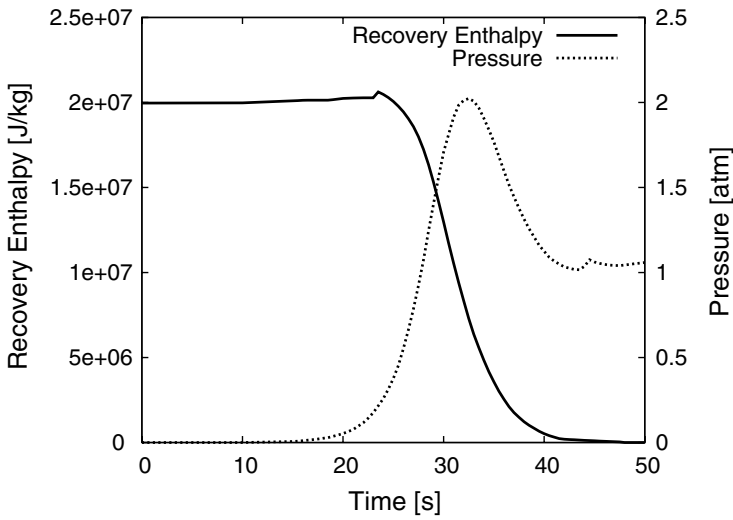

a) Recovery enthalpy and pressure numerical treatment, as well as numerical validations, is described in greater detail in [2-4]. In addition to the improvement discussed in this paper, the present code also takes into account different coordinate systems (cylindrical and spherical), and allows ablation on both sides of the domain, using a new tridiagonal solver [5].

\section{B. Model Validation}

All the numerical validations presented in [2-4] are performed successfully on the code (grid refinement studies, nonlinear convergence studies, boundary conditions verifications, and timing studies). Doing so insures that MOPAR is coded properly and that the expected convergence behavior is achieved (second order in space and first order in time). Because these results are exactly the same as those published in the literature, and because they serve no purpose in the context of this paper, they are omitted here. Two other validations are, however, presented: they demonstrate the validity of the code as a model.

\section{Code-to-Code Comparison}

The first validation compares the present code to the one developed at Sandia National Laboratories [1-4], which was extensively compared to the old charring materials ablation (CMA) code [6] as well as Sandia's one-dimensional direct and inverse thermal (SODDIT) code [7]. The simulation is performed using solid and gas properties given in [2]. The reentry conditions used on the ablative surface, shown in Fig. 1, are also exactly those presented in [2], and were generated using a series of reentry codes at Sandia National Laboratories (2IT [8], SANDIAC [9,10], and HIBLARG [11]). The discontinuities in the quantities are caused by the numerical scheme used to model the transition to turbulence. For this particular comparison, hot wall and blowing corrections are not applied to the incident aeroheating flux; thermal-radiation cooling is also omitted. The thermochemical table used to interpolate the rates of ablation is generated using ACE-SNL [12], and is identical to the one presented in [2]. The calculations are performed on a 75 element logarithmic grid, with a 1.05 progression, over a $1.27 \mathrm{~cm}$ domain, using a time step of $0.2 \mathrm{~s}$.

Figure 2 presents the comparison of results for surface temperature and surface recession for a carbon/phenolic ablator, and surface temperature and recession rate for a carbon/carbon ablator. As expected, the plotted quantities from both simulations agree well (the highest discrepancies are less than $8 \%$ on temperature and less than $1 \%$ on the surface recession for carbon/phenolic; the results are essentially identical for carbon/carbon, with less than $1 \%$ of errors everywhere). The very small discrepancies are due to a different method used to interpolate the material properties of the ablator.

\section{Experimental Results}

The second validation presented here compares the code to two experiments [13]. For these test cases, a $1.27-\mathrm{cm}$-thick carbonphenolic material was exposed to an air arcjet flow for which the

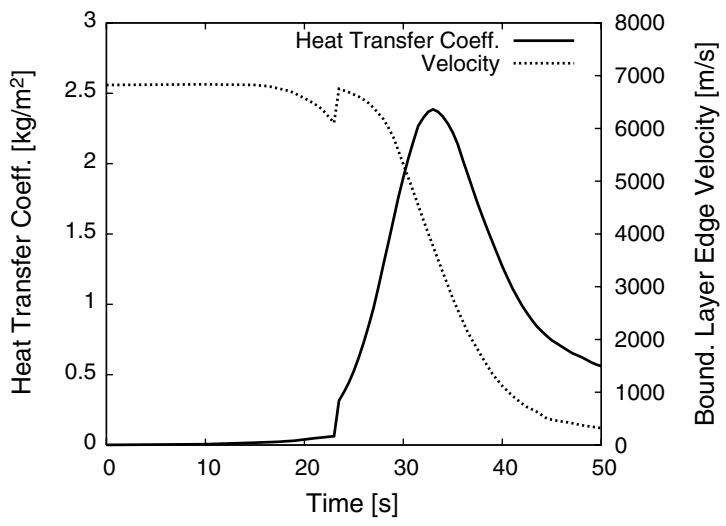

b) Heat transfer coefficient and velocity

Fig. 1 Reentry conditions for the first validation (the discontinuity on each curve is caused by the transition to turbulent flow). 


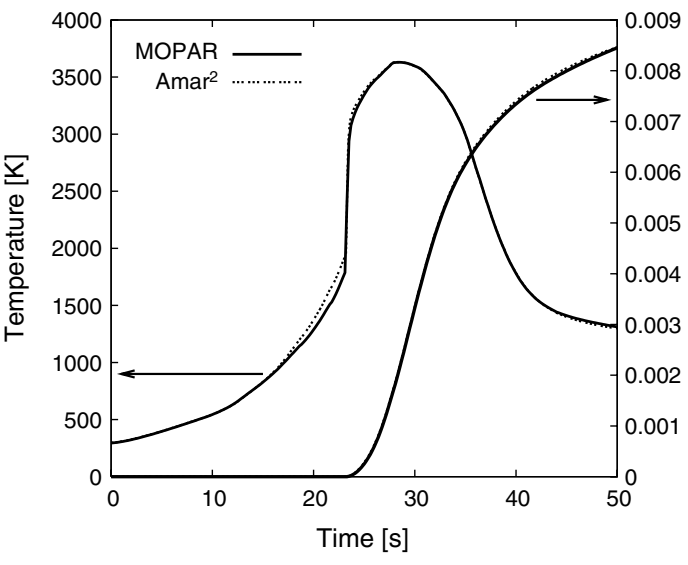

a) Charring ablator

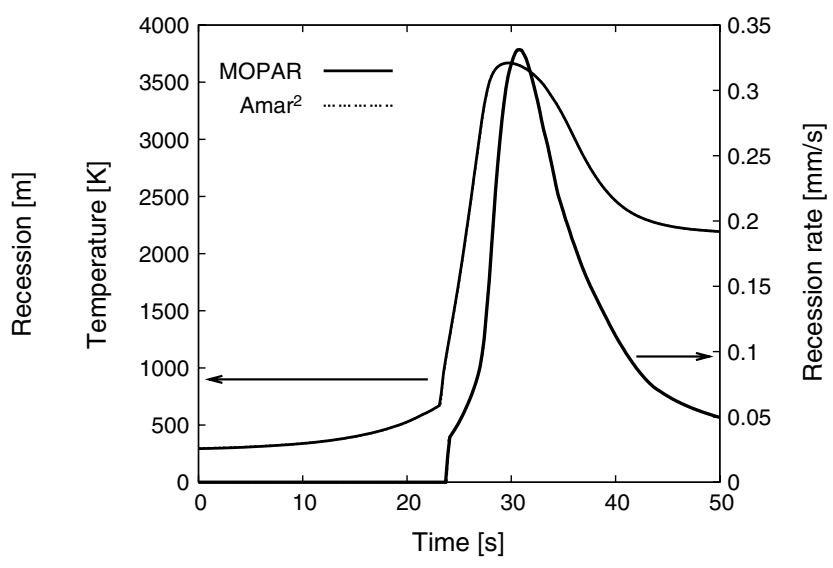

b) Carbon/Carbon ablator

Fig. 2 Comparison of surface temperature and surface recession between [2] and present work for ablation during reentry (arrows link the curves to their respective axes).

parameters are listed in Table 1 . The calculations are performed on a 150 element logarithmic grid, with a 1.01 progression, using a constant time marching of $0.1 \mathrm{~s}$. A blowing correction as well as a hot wall correction is applied to the incident heat flux. The properties used in this simulation are the same as those given in [13]. However, because this reference only gives the pyrolysis gas specific heat at constant pressure, the values of the molar mass, enthalpy, both specific heats, and viscosity from [14] are used instead. Finally, the porosity $\phi$ and the permeability $K$ of the test piece are taken from [2], as is the generic thermochemical carbon-phenolic ablation table.

The graphs of Fig. $\underline{3}$ show the results for surface and back temperature, surface recession, and pyrolysis front recession. Comparisons are included to both the experimental and numerical results published by Sutton [13]. For both test cases, the computer predictions obtained with the present model are considered within the acceptable ranges of the experimental results.

Because the exact method used to experimentally measure the pyrolysis front is not described, a value of $50 \%$ of the resin initial density is chosen as the approximation of the char/virgin interface. This choice is justified by the fact that the pyrolysis front is quite steep, as illustrated in Fig. 4, and that Arrhenius-type relations are used to describe the chemical decomposition, therefore ruling out using $0 \%$ as a criterion. As mentioned in [13], the discrepancy between the simulation and the experimental results, as far as the recession rates are concerned, is caused by the fact that the cool-down period is not taken into account. As in [13], it was observed that in both cases, the pyrolysis front keeps moving, reaching the back surface at approximatively $75 \mathrm{~s}$ for the first test case, and $42 \mathrm{~s}$ for the second one. In reality, the test pieces were quenched by argon right after the arcjet exposure, in an attempt to stop the chemistry as soon as possible. Because no exact description of the process is available, it is not possible to produce an accurate cool-down model.

The discrepancy for the surface ablation front is, obviously, not caused by the cool down because ablation immediately stops after exposure, and the simulations overpredict the distance. The difference can be attributed to two reasons: first, the empirical thermochemical tables used are not generated for the specific carbonphenolic material of the experiment (Narmco 4028). Also, thermal expansion of the test pieces was observed and is not included in the present model. Depending on the type of material, as well as the fiber orientation, this process may not necessarily be reversible, thus

Table 1 Test conditions for the heat transfer blowing correction verification

\begin{tabular}{lcccc}
\hline \hline Test case & $H_{\mathrm{st}},[\mathrm{J} / \mathrm{kg}$ & $q_{\mathrm{st}}, \mathrm{W} / \mathrm{m}^{2}$ & $p_{\mathrm{st}}, \mathrm{Pa}$ & $\Delta t, \mathrm{~s}$ \\
\hline 1 & $11.60 \times 10^{6}$ & $1.44 \times 10^{6}$ & 0.07 & 60 \\
2 & $26.65 \times 10^{6}$ & $7.05 \times 10^{6}$ & 0.32 & 35.0 \\
\hline \hline
\end{tabular}

causing variations in the thickness that could potentially affect the ablation front measurements.

In addition to the reasons just described, the discrepancies could be explained by the omission of other physical phenomena in the model: carbon fiber orientation, delamination (crack formation), and spallation (mechanical ablation). All of these effects can lead to significant differences in the results. The difference in results can also be attributed to the generic carbon-phenolic thermochemical table, as well as a slightly different pyrolysis gas. Finally, the experimental uncertainties for these test cases are unknown, meaning that the appreciation of the results cannot be assessed. Because of all of these factors, the presented numerical results are considered to agree fairly well with the experimental data.

\section{Kinetic Energy}

For the governing equations to be more rigorous, the kinetic energy should be included in the mixture energy equation, Eq. (1). Kinetic energy is added in the time-dependent energy content term (first term), the gas flux term (second term), and the grid convection term (fourth term). The contributions in the control volume are as follows:

Energy content term:

$$
\tilde{E}_{\text {kin }}=\frac{\mathrm{d}}{\mathrm{d} t} \int_{\mathrm{cv}} \rho \frac{v_{g}^{2}}{2} \mathrm{~d} V
$$

Gas flux term

$$
\dot{G}_{\text {kin }}=\int_{\text {cs }} \phi \rho_{g} \frac{v_{g}^{2}}{2} v_{g} \mathrm{~d} A
$$

Grid convection term

$$
\dot{E}_{\text {kin }}=-\int_{\text {cs }} \rho \frac{v_{g}^{2}}{2} v_{\mathrm{cs}} \mathrm{d} A
$$

In the reentry test case described in the previous section, the addition of these terms has little to no effect on the final results, thus validating their omission in most thermal response codes (CMA [6], Sandia National Laboratories [3], FIAT [15]; however SCMA [1] ], in its latest version, uses these contributions). As seen in Fig. 5, the difference between the kinetic terms and the other terms is several orders of magnitude. It is to be noted that depending on the charring material used, these effects may start to appear. A material with a very high rate of decomposition, as well as high porosity, may lead to higher gas velocity. With a simple correlation using, for instance, the velocity shown in Fig. 8d, it is possible to estimate that a gas velocity of the order of $100 \mathrm{~m} / \mathrm{s}$ (i.e., an increase of 2 orders of magnitude) would result in the contributions shown in Fig. $\underline{5 b}$ to register in Fig. 5a. 


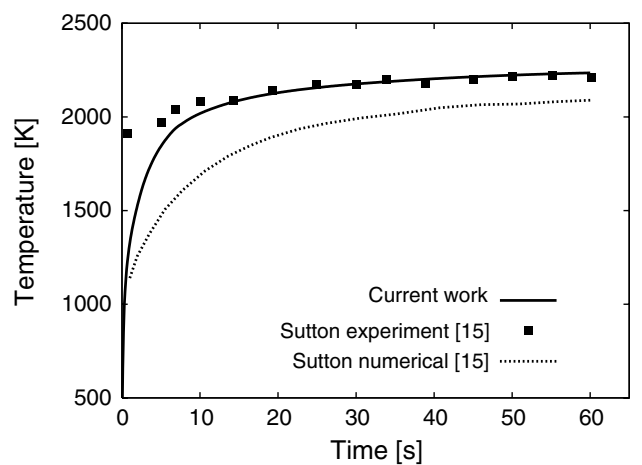

a) Surface temperature for test-case 1

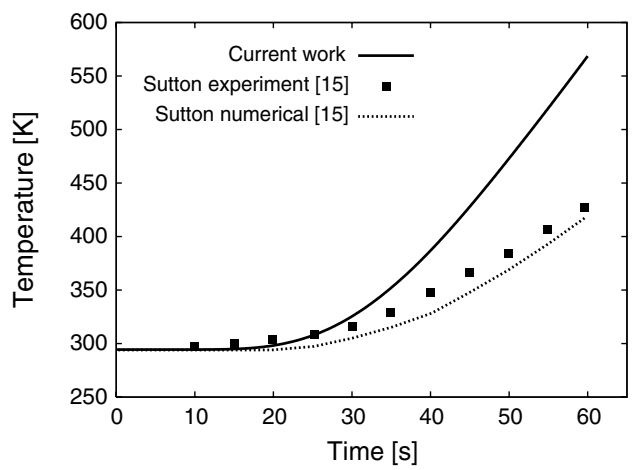

c) Back face temperature for test-case 1

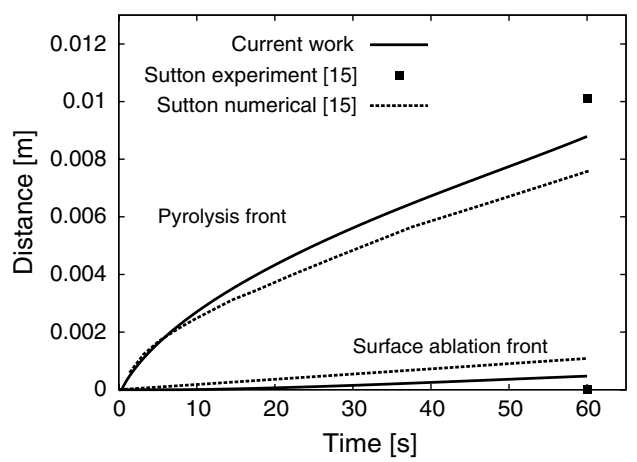

e) Surface ablation and pyrolysis front for test-case 1

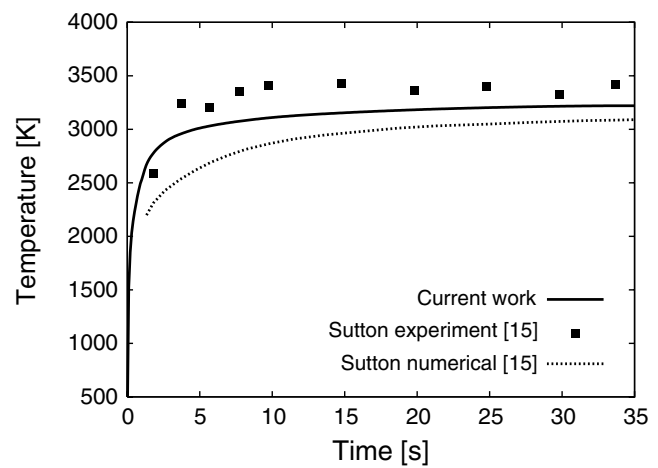

b) Surface temperature for test-case 2

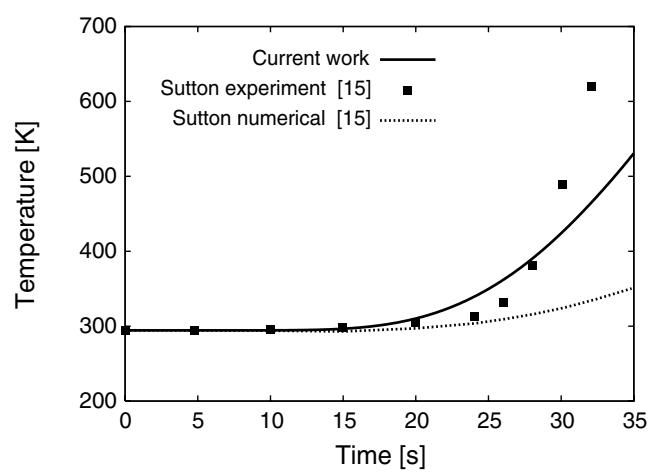

d) Back face temperature for test-case 2

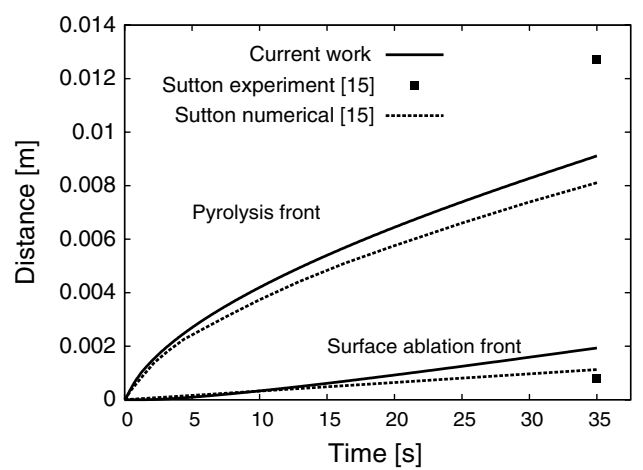

f) Surface ablation and pyrolysis front for test-case 2

Fig. 3 Comparisons of the present code to experimental results.

\section{Forchheimer's Law}

1. Forchheimer Number as a Criterion

In other works, either Darcy's law [3] or Forchheimer's law [6,16] is used to calculate the velocity of the gas within the ablator. The decision to use one law or the other is usually linked to the Reynolds number. As pointed out in [17], there is enormous disagreement on the conditions where a flow is not described by Darcy's equation, mainly because the characteristic length is difficult to measure or evaluate. The two main lengths used in the literature are the pore length and the particle size. However, because both of these quantities are defined on the microscopic scale, it is inappropriate to use them to model macroscopic effects without a careful averaging based on the exact structure of the material. For instance, these quantities do not take into account the state of the porosity of the material, more specifically the tortuosity (how convoluted are the channels or pores) and the closed porosity (unconnected "bubble"). They also cannot account for the fact that the gas flow may be subject to recirculation zones. Therefore, simply using the pore size to evaluate the flow type does not account for these phenomena.

To address this problem, a modified formulation of Forchheimer's law was proposed [18]. In Cartesian coordinates, this equation is

$$
\frac{\partial p}{\partial x}=-\frac{\mu}{K} v_{g}^{\prime}(1+F o)
$$

where $F o$, the Forchheimer number, is given by

$$
F_{o}=\frac{\beta K \rho v_{g}^{\prime}}{\mu}
$$

This number indicates when microscopic effects (pore size) are perceivable at a macroscopic (geometry size) level. In this formulation, it is easy to see that when $F o \ll 1$, the equation simply reduces to Darcy's law [Eq. (5)]. Therefore, it is more logical to use the Forchheimer number to predict non-Darcian flow, and thus more rigorous to use Forchheimer's law in the model.

The law can be derived from the momentum equation [Eq. (4)], using averaging methods [19]:

$$
\rho\left(\frac{\partial v_{g}}{\partial t}+v_{g} \frac{\partial v_{g}}{\partial x}\right)+\frac{\partial p}{\partial x}=\mu \frac{\partial^{2} v_{g}}{\partial x^{2}}
$$




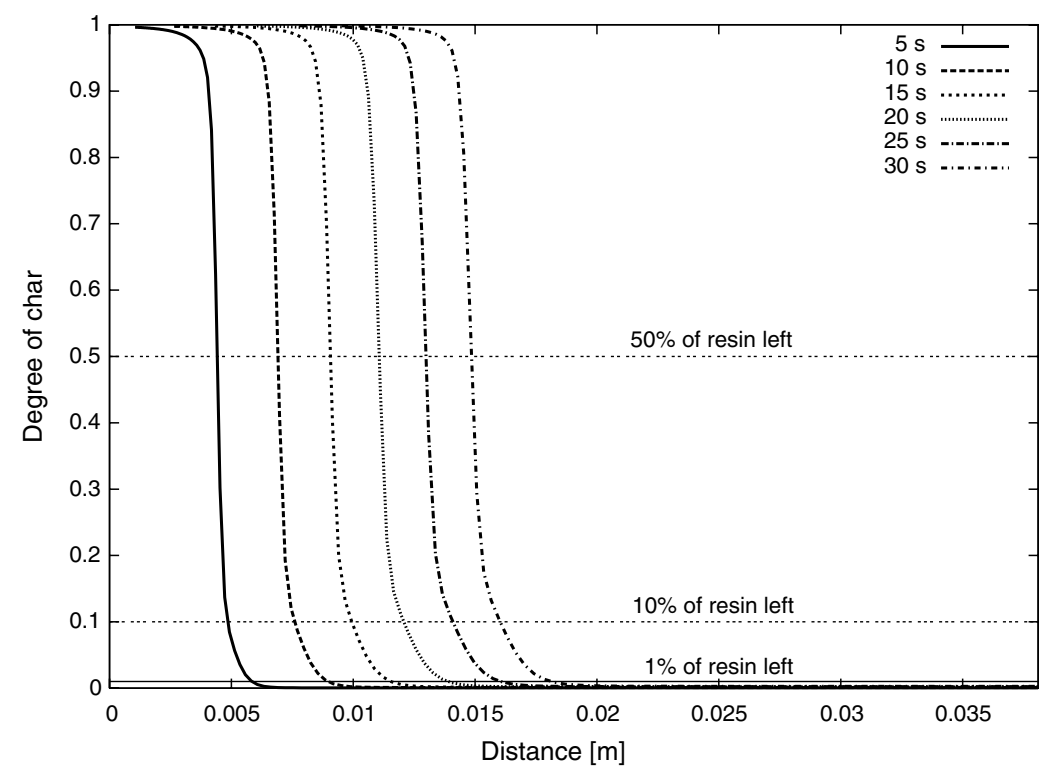

Fig. 4 Evolution of the pyrolysis (char) front for the second test case. The degree of char is obtained using the ratio of ablated resin density to initial resin density $\left(\rho_{v}-\rho_{s}\right) /\left(\rho_{v}-\rho_{c}\right)$.

One can also obtain the same results by simply dimensionalizing the momentum equation, replacing the spatial derivative by the inverse of the square root of the permeability $K$ and the velocity by the superficial velocity $v_{g}^{\prime}$.

The main obstacle in using Forchheimer's law is a lack of knowledge of parameter $\beta$; over the years, many attempts to characterize this parameter have been made. In 1952, Ergun [20] used this formulation to model a flow through a packed bed, linking $K$ and $\beta$ to the porosity $\phi$ and the characteristic diameter of the particles $D$, by rewriting Eq. (ㅁ) as

$$
\frac{\Delta p}{\Delta x}=150 \frac{(1-\phi)^{2}}{D^{2} \phi^{3}} \mu v^{\prime}+1.75 \frac{(1-\phi)}{D \phi^{3}} \rho v^{\prime 2}
$$

Because this model has been demonstrated as inadequate in many applications, many attempts were made to adapt this formulation by changing the definition of the characteristic diameter $D$ (using pore size, for instance) [21,22], with limited success. This can be explained by the fact that a macroscopic quantity is being linked to a microscopic quantity. Nevertheless, it is still possible to use the equation in another fashion. By comparing Eqs. (ㅁ) and (13), it can be assumed that

$$
K=\frac{D^{2} \phi^{3}}{150(1-\phi)^{2}}
$$

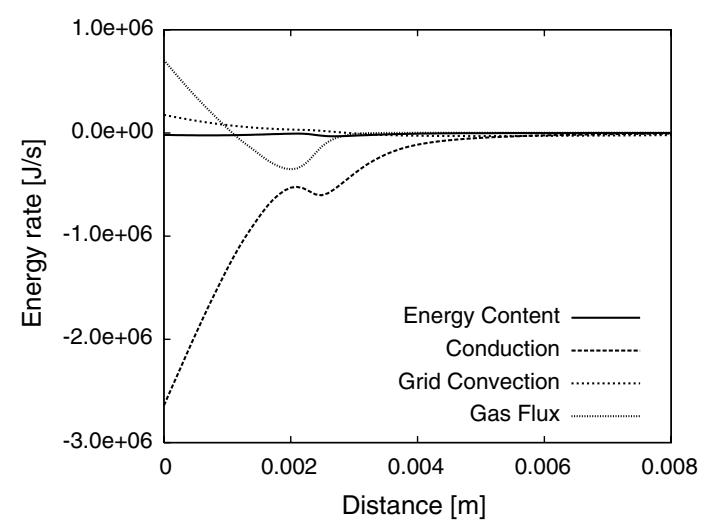

a) All terms, except the kinetic terms and Ergun's formulation can therefore be written as

$$
\frac{\Delta p}{\Delta x}=\frac{\mu v^{\prime}}{K}+\frac{1.75}{\sqrt{150 \phi^{3}}} \frac{1}{\sqrt{K}} \rho v^{\prime 2}
$$

which gives a definition of the $\beta$ parameter:

$$
\beta=\frac{\beta_{K}}{\sqrt{K}}=\frac{1}{7} \frac{1}{\phi^{3 / 2}} \frac{1}{\sqrt{K}}
$$

Equation (14) is of interest because it does not require a microscopic parameter $(\bar{D})$, and only relies on the macroscopic parameter $K$. This approach can therefore give a reasonable approximation for $\beta$. Using the generic carbon-phenolic values employed in the previous test case, taken from [2], it is possible to evaluate $\beta$ : those values are presented in Table 2 .

It is also interesting to note that if $\beta$ is defined by Eq. (15), the Forchheimer number becomes

$$
F o=\beta_{K} \frac{\sqrt{K} \rho\left|v_{g}^{\prime}\right|}{\mu}
$$

which is the Reynolds number defined with a characteristic length of $\sqrt{K}$ and multiplied by $\beta_{K}$. Considering that Forchheimer's law can be derived by nondimensionalizing the momentum equation using

Fig. 5 Energy rates of each contributing term of the mixture energy equation, taken at $t=25 \mathrm{~s}$ of the trajectory. 
Table 2 Estimation of $\beta$ and $\beta_{K}$ for carbon phenolic using Ergun's equation

\begin{tabular}{lcccc}
\hline \hline Extent of reaction & Porosity $(\phi)$ & Permeability $(K), \mathrm{m}^{2}$ & $\beta, \mathrm{m}^{-1}$ & $\beta_{K}$ \\
\hline 0.00 & 0.010 & $5.00 \times 10^{-20}$ & $6.39 \times 10^{11}$ & 142.0 \\
0.25 & 0.095 & $3.00 \times 10^{-18}$ & $2.82 \times 10^{9}$ & 4.88 \\
0.50 & 0.180 & $3.00 \times 10^{-17}$ & $3.42 \times 10^{8}$ & 1.87 \\
0.75 & 0.265 & $2.00 \times 10^{-17}$ & $2.34 \times 10^{8}$ & 1.05 \\
1.00 & 0.350 & $1.00 \times 10^{-13}$ & $2.18 \times 10^{6}$ & 0.689 \\
\hline \hline
\end{tabular}

Table 3 Experimental and estimated values for $\beta$ and $\boldsymbol{\beta}_{K}$ for various porous rocky materials [17]

\begin{tabular}{lcccccc}
\hline \hline Material & Porosity $(\phi)$ & Permeability $(K), \mathrm{m}^{2}$ & $\beta$ (exp.), $\mathrm{m}^{-1}$ & $\beta_{K}$ (exp.) & $\beta$ (est.), $\mathrm{m}^{-1}$ & $\beta_{K}$ (est.) \\
\hline Dakota sandstone & 0.14 & $3.48 \times 10^{-15}$ & $1.5788 \times 10^{10}$ & 931.36 & $4.6230 \times 10^{7}$ & 2.7272 \\
Indiana limestone & 0.15 & $2.16 \times 10^{-14}$ & $3.600 \times 10^{9}$ & 529.09 & $1.6732 \times 10^{7}$ & 2.4590 \\
Berea sandstone & 0.18 & $1.96 \times 10^{-13}$ & $2.88 \times 10^{8}$ & 127.50 & $4.2254 \times 10^{6}$ & 1.8707 \\
\hline \hline
\end{tabular}

Table 4 Experimental and estimated values for $\boldsymbol{\beta}$ and $\boldsymbol{\beta}_{K}$ for various porous ceramic foams [21]

\begin{tabular}{lccccc}
\hline \hline Porosity $(\phi)$ & Permeability $(K), \mathrm{m}^{2}$ & $\beta$ (exp.), $\mathrm{m}^{-1}$ & $\beta_{K}$ (exp.) & $\beta$ (est.), $\mathrm{m}^{-1}$ & $\beta_{K}$ (est.) \\
\hline 0.85 & $(0.39 \pm 0.02) \times 10^{-8}$ & $(1.00 \pm 0.03) \times 10^{4}$ & $0.625 \pm 0.016$ & $2.92 \times 10^{3}$ & 0.182 \\
0.85 & $(0.51 \pm 0.12) \times 10^{-8}$ & $(5.6 \pm 2.2) \times 10^{3}$ & $0.397 \pm 0.047$ & $2.55 \times 10^{3}$ & 0.182 \\
0.88 & $(2.56 \pm 1.00) \times 10^{-8}$ & $(1.18 \pm 0.15) \times 10^{3}$ & $0.188 \pm 0.037$ & $1.08 \times 10^{3}$ & 0.173 \\
0.89 & $(3.20 \pm 2.38) \times 10^{-8}$ & $(1.587 \pm 0.076) \times 10^{3}$ & $0.28 \pm 0.11$ & $9.51 \times 10^{2}$ & 0.170 \\
\hline \hline
\end{tabular}

Table 5 Experimental and estimated values for $\beta$ and $\beta_{K}$ for various porous carbon foams [23]

\begin{tabular}{lcccccc}
\hline \hline Material & Porosity $(\phi)$ & Permeability $(K), \mathrm{m}^{2}$ & $\beta$ (exp.) $\left[\mathrm{m}^{-1}\right]$ & $\beta_{K}$ (exp.) & $\beta$ (est.), $\mathrm{m}^{-1}$ & $\beta_{K}$ (est.) \\
\hline $219-3$ & 0.86 & $2.41 \times 10^{-10}$ & $4.7951 \times 10^{4}$ & 0.7444 & $1.1538 \times 10^{4}$ & 0.1791 \\
$218-3$ & 0.88 & $4.46 \times 10^{-10}$ & $2.1535 \times 10^{4}$ & 0.4548 & $8.1943 \times 10^{3}$ & 0.1731 \\
POCO $^{\mathrm{TM}}$ & 0.82 & $6.13 \times 10^{-10}$ & $1.8002 \times 10^{4}$ & 0.4457 & $7.7705 \times 10^{3}$ & 0.1924 \\
\hline \hline
\end{tabular}

$\sqrt{K}$ as the characteristic length, as mentioned earlier, this is not too surprising.

Although no measurements for $\beta$ of TPS materials have been published in the literature, it has been for various other materials. Using these values, it is possible to verify the extent of the validity of Ergun's equation. Tables $\underline{3}-\underline{5}$ show various measured values for other types of solid materials (i.e., not packed material), found in the literature. From these data, it is possible to compare with the values obtained from Ergun's equation. For materials that can be compared to pyrolyzing TPS materials (Tables 4 and 5), both values are of the same order of magnitude; some of them are even within the experimental uncertainty. However, for dissimilar materials, such as the ones presented in Table $\underline{3}$, the discrepancy is 2 orders of magnitude. At this point, it is difficult to say if that difference is due to the small porosity value or to the type of porosity (heavy tortuosity and closed porosity) of such materials.

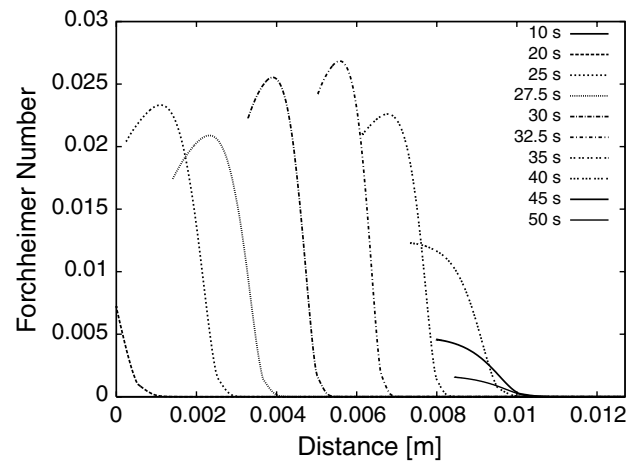

a) Forchheimer number at various times during the reentry simulation, using $\beta=1.222 / \sqrt{K}$
Ahn et al. in [16] obtained $\beta_{K}=1.222$ for carbon phenolic using the empirical model devised by Ward [24], which simply removes the direct dependency on the pore size by setting a constant value for $\beta_{K}$. Ward tabulated this parameter for multiple types of granular material (sand, glass beads, gravel, and granular coal), and used water as the fluid. Because this type of solid material does not include the concept of closed porosity and tortuosity, and that water behaves very differently than pyrolysis gas, it is hard to adapt that methodology to a carbon-phenolic material. Nevertheless, the value proposed by [16] is of the same order of magnitude as the value obtained using Ergun's equation (Table 2).

Even though the modified Ergun approach can give estimates of $\beta$ and $\beta_{K}$, an experimental approach would give better results. An effective value could be obtained using the setup and method presented in [17]. With this methodology, already published experimental data $[25,26]$ could easily be employed to calculate $\beta$ for

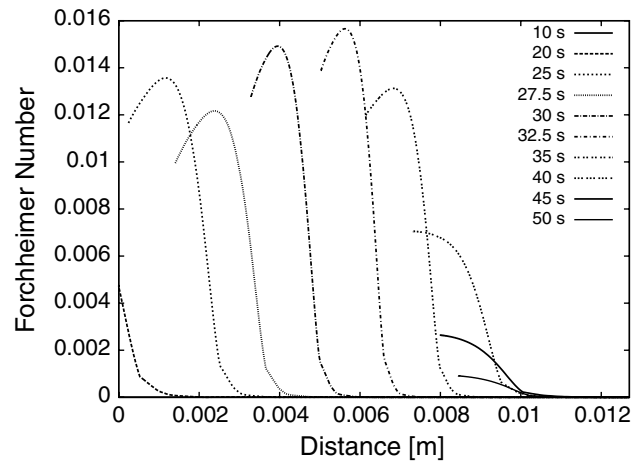

b) Forchheimer number at various times during the reentry simulation, using $\beta$ calculated with Eq. 15

Fig. 6 Forchheimer number for the generic reentry simulation of Sec. II.B. 


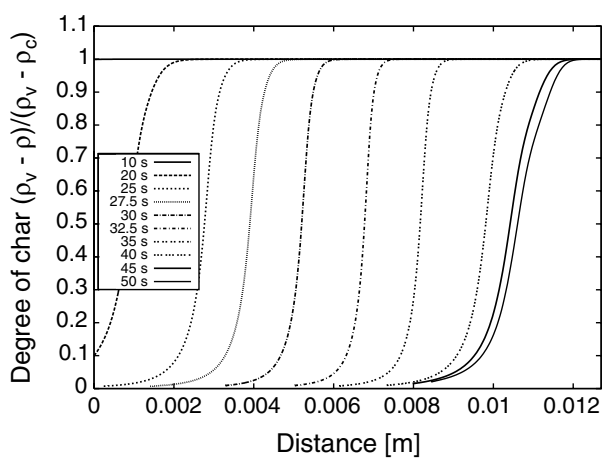

a) Degree of char $\left(\left(\rho_{v}-\rho_{s}\right) /\left(\rho_{v}-\rho_{c}\right)\right)$ at various times during the reentry simulation, using $\beta$ calculated with Eq. 15

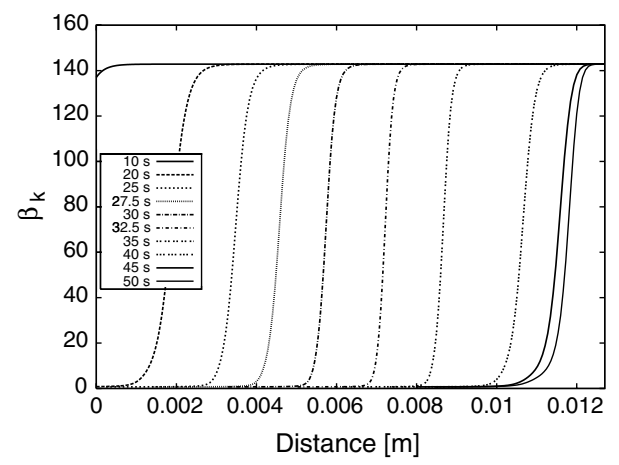

b) $\beta_{K}$ at various times during the reentry simulation, using $\beta$ calculated with Eq. 15

Fig. 7 Degree of char and $\beta_{K}$ for the generic reentry simulation of Sec. II.B.

two frequently used charring ablators, the phenolic impregnated carbon ablator (PICA) and the silicone impregnated reusable ceramic ablator (SIRCA). However, other experimental values, notably the length of the test pieces and pressure at both ends, would have to be known.

Finally, it is also important to point out that the deviation from Darcy's law presented in [25], the so-called Klinkenberg model, is not in any way related to the effects contained in Forchheimer's law. Although the latter takes into account high velocity effects and the type of porosity, the former models the slip-flow behavior of the gas within porous media. The relation used to calculate permeability with Klinkenberg effects is

$$
K=K_{0}(1+b / p)
$$

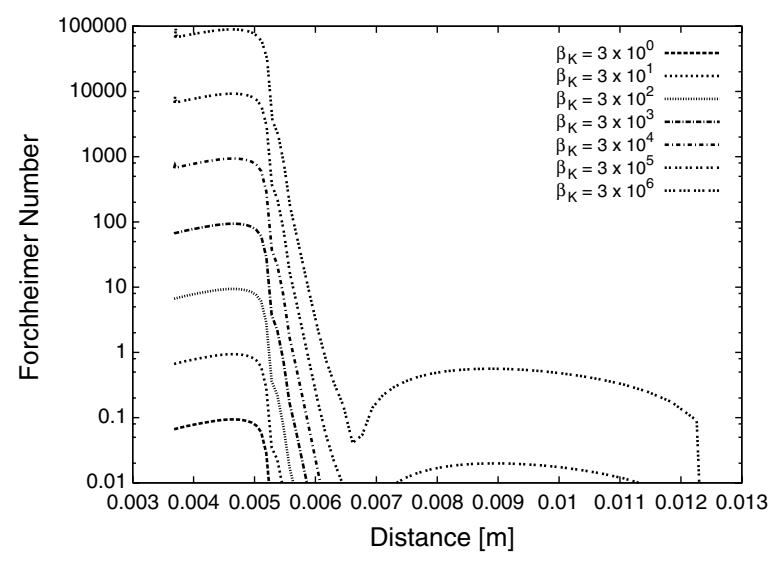

a) Forchheimer number

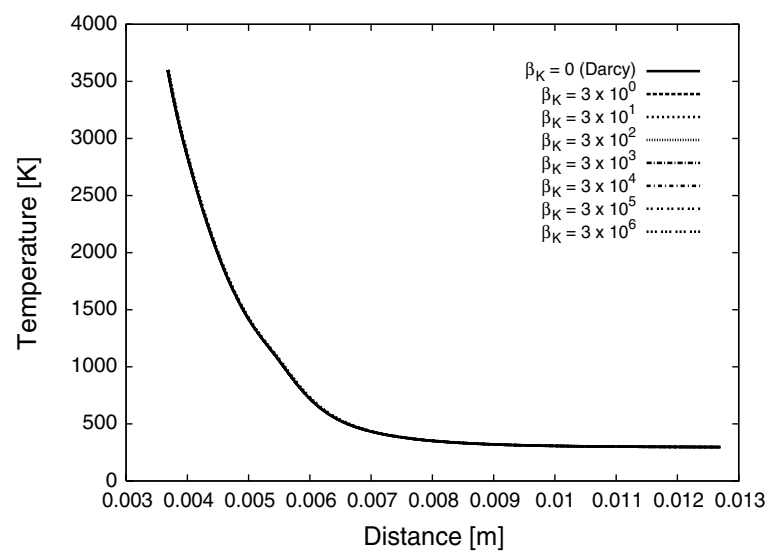

c) Temperature
In this equation, permeability coefficient $b$ is a function of the gas composition, unlike $K_{0}$, the permeability in the no-slip regime, which is only material dependent. It is also to be noted that both these parameters are also found experimentally, and are therefore effective values, essentially valid for the conditions of the experiment.

\section{Effects of $\beta$}

To assess the influence of using Forchheimer's law instead of Darcy's, a study is performed using the test case presented in Sec. II.B. First, the simulation is run using a constant value of $\beta_{K}=1.222$, as suggested in [16]; the resulting values for the Forchheimer number are presented in Fig. 6a. The same test case is also run using Eq. (15) to compute $\beta_{K}$; the results are presented in Fig. 6b. For both cases, the value of $F o$ is of the order of 0.01 ; by

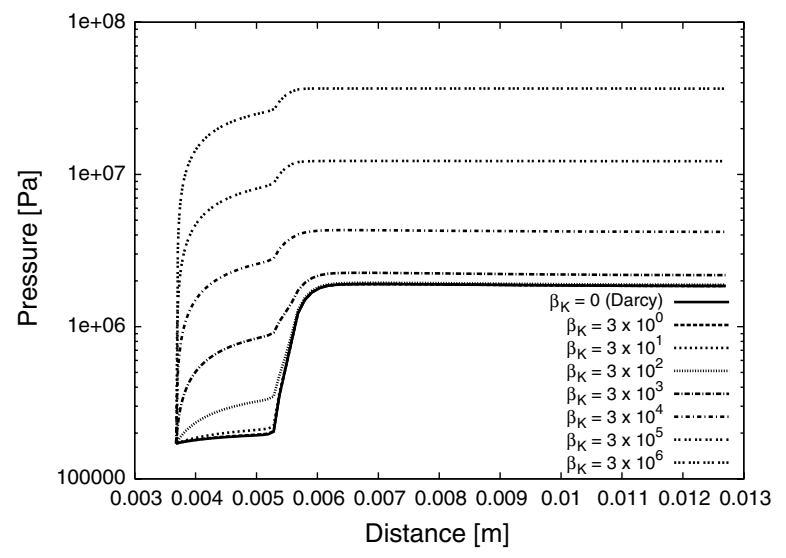

b) Pressure

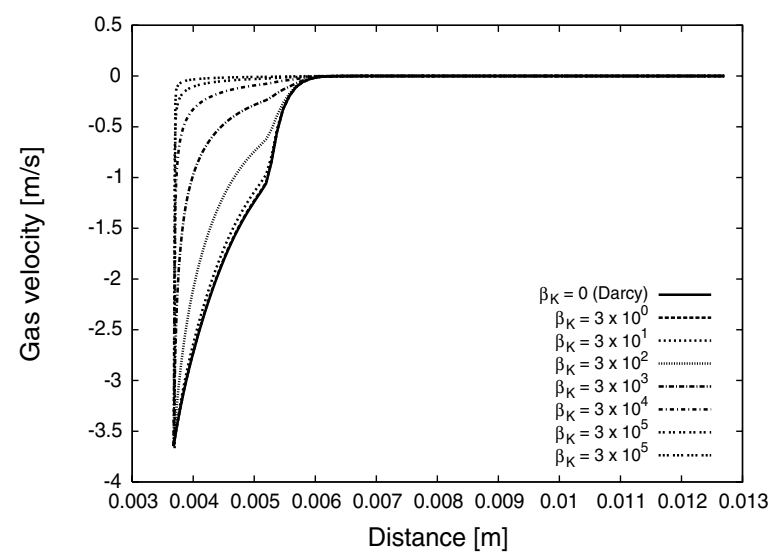

d) Velocity

Fig. 8 Influence of inertial parameter $\beta_{K}$ on the internal gas behavior: $\beta_{K}$ varies from 0 to $3 \times 10^{6}$. 
inserting this value in Eq. (10), it can be seen that the effects, although small, are not necessarily negligible. From these figures, we can also see that approximating $\beta_{K}$ to a constant value, based on the charred properties is not a bad assumption because, as can be seen in Fig. 7a, where the degree of char is presented, the pyrolysis region occurs in a very narrow region (the so-called pyrolysis front). Therefore, most of the region of interest, i.e., where pyrolysis gas is present and has an appreciable velocity, is where the material is completely charred: this is illustrated in Fig. $7 \mathrm{~b}$ by presenting the values of $\beta_{K}$ at various times during the simulation.

From these results, it is safe to conclude that in thermodynamic conditions where pyrolysis occurs over a very narrow distance, a constant value of $\beta$, based on experimental measurements of a completely charred specimen, would be satisfactory.

\section{Parametric Study}

Although many studies have been performed [27-29] on the behavior of pyrolysis gas, none account for the possibility of $\beta$ being a significant factor. A simple parametric study allows one to see that even though there is little to no effect on the temperature of the material, other quantities such as velocity, density and, obviously, pressure are greatly affected. The graphs of Fig. 8 show the behavior of the pyrolysis gas at the $30 \mathrm{~s}$ reentry point of the generic trajectory used in the previous section. It can clearly be seen that as soon as the Forchheimer number becomes greater than $1\left(\beta_{K} \sim 10\right)$, notable effects appear (the lines no longer overlapping each other). Given that spallation has been observed [13] in many materials considered for reentry systems, it is therefore important to take these effects into account. It is clear that such a high pressure zone as the one that appears near the wall-flow boundary will cause spallation. That being said, however, large $\beta_{K}$ is, there is no discernible change in the temperature. This is easily explained by the fact that the model assumes that the gas and the solid are in equilibrium, and that, as demonstrated in Sec. II.C, the kinetic energy has little influence on the energy contribution.

\section{Conclusions}

To improve heat and ablation rate modeling on reentry vehicles, a one-dimensional material response model that includes pyrolysis and surface ablation has been presented. The code was extensively verified, and has been successfully compared to experimental data, as well as with a similar code. The new developments provide a more precise analysis of the contribution of the pyrolysis gas to the behavior of the TPS. More specifically, the use of the Forchheimer number as an indicator of non-Darcian flow behavior, instead of the average pore-size Reynolds number, has been highlighted. The use of that parameter removes the dependency on microscopic properties and allows the macroscopic properties of the TPS material, such as closed porosity and tortuosity, to be taken into account. Using Ergun's equation, a simple way to calculate the Forchheimer number was presented, and a simple test case was modeled using that value. Finally, to evaluate the effects of that parameter on the thermodynamics properties, a parametric study using a simple reentry trajectory with a generic carbon-phenolic ablator, and the conditions necessary to observe a non-Darcian flow were described. Even though the inclusion of the new model does not significantly affect the temperature, the variations in inner pressure observed might lead to other phenomena, such as spallation.

\section{Acknowledgments}

The authors would like to thank the Government of Québec which, through the Fonds de recherche sur la nature et les technologies, provides a fellowship to the first author. Additional funding is provided by the Constellation University Institutes Program, under NASA Grant NCC3-989. The authors would also like to thank Adam J. Amar of NASA Johnson Space Center and formerly from Sandia National Laboratories, for numerous insightful discussions.

\section{References}

[1] Blackwell, B. F., and Hogan, R. E., "One-Dimensional Ablation Using Landau Transformation and Finite Control Volume Procedure," Journal of Thermophysics and Heat Transfer, Vol. 8, No. 2, April-June 1994, pp. 282-287.

doi: $10.2514 / 3.535$

[2] Amar, A. J., "Modeling of One-Dimensional Ablation with Porous Flow Using Finite Control Volume Procedure," M.S. Thesis, North Carolina State University, Raleigh, NC, 2006.

[3] Amar, A. J., Blackwell, B. F., and Edwards, J. R., "One-Dimensional Ablation Using a Full Newton's Method and Finite Control Volume Procedure," Journal of Thermophysics and Heat Transfer, Vol. 22, No. 1, Jan. 2008, pp. 72-82. doi: $10.2514 / 1.29610$

[4] Amar, A. J., Blackwell, B. F., and Edwards, J. R., "Development and Verification of a One-Dimensional Ablation Code Including Pyrolysis Gas Flow," Journal of Thermophysics and Heat Transfer, Vol. 23, No. 1, Jan.-March 2009, pp. 59-71. doi: $10.2514 / 1.36882$

[5] Martin, A., and Boyd, I. D., "Variation of the Thomas Algorithm for Opposed-Border Tridiagonal Systems of Linear Equations," Соттиnications in Numerical Methods in Engineering, 2008. doi:10.1002/cnm.1172

[6] Kendall, R. M., Rindal, R. A., and Bartlett, E. P., "Thermochemical Ablation," AIAA Paper 1965-642, 13-15 Sept. 1965.

[7] Blackwell, B. F., Douglas, R. W., and Wolf, H., "A User's Manual for the Sandia One-Dimensional Direct and Inverse Thermal (SODDIT) Code," Sandia National Laboratories TR SAND 85-2478, 1987.

[8] Brant, D., Wade, M., and Moran, J., "Computational Technique for Three-Dimensional Inviscid Flow Fields About reentry Vehicles: Volume II, User's Manual," Air Force Space and Missile Systems Organization TR SAMSO TR-79-5, DIN: 78SDR2176, April 1978.

[9] Noack, R. W., and Lopez, A. R., "Inviscid Flow Field Analysis of Complex Reentry Vehicles: Volume I, Description of Numerical Methods," Sandia National Laboratories TR SAND 87-0776/1, 1988.

[10] Noack, R. W., and Lopez, A. R., "Inviscid Flow Field Analysis of Complex Reentry Vehicles: Volume II, User's Manual," Sandia National Laboratories TR SAND 87-0776/2, 1988.

[11] Polansky, G. F., "Hypersonic Integral Boundary Layer Analysis of Reentry Geometries (HIBLARG) Code Description and User's Manual Ver. 2.0," Sandia National Laboratories TR SAND 89-0552, 1990.

[12] Powars, C. A., and Kendall, R. M., Aerotherm Chemical Equilibrium (ACE) Computer Program - User's Manual, Aerotherm Corporation, Mountain View, CA, May 1969.

[13] Sutton, K., "An Experimental Study of a Carbon-Phenolic Ablation Material," NASA Langley Research Center TN D-5930, 1970.

[14] Wakefield, R. M., and Pitts, W. C., "Analysis of the Heat-Shield Experiment on the Pioneer-Venus Entry Probes," AIAA Paper 19801494, 14-16 July 1980.

[15] Chen, Y.-K., and Milos, F. S., "Ablation and Thermal Response Program for Spacecraft Heatshield Analysis," Journal of Spacecraft and Rockets, Vol. 36, No. 3, May-June 1999, pp. 475-483. doi: $10.2514 / 2.3469$

[16] Ahn, H.-K., Park, C., and Sawada, K., "Response of Heatshield Material at Stagnation Point of Pioneer-Venus Probes," Journal of Thermophysics and Heat Transfer, Vol. 16, No. 3, July-Sept. 2002, pp. 432-439. doi: $10.2514 / 2.6697$

[17] Zeng, Z., and Grigg, R., "A Criterion for Non-Darcy Flow in Porous Media," Transport in Porous Media, Vol. 63, No. 1, 2006, pp. 57-69. doi:10.1007/s11242-005-2720-3

[18] Ruth, D., and Ma, H., "On the Derivation of the Forchheimer Equation by Means of the Averaging Theorem," Transport in Porous Media, Vol. 7, No. 3, 1992, pp. 255-264. doi:10.1007/BF01063962

[19] Whitaker, S., "Flow in Porous Media 1: A Theoretical Derivation of Darcy's law," Transport in Porous Media, Vol. 1, No. 1, 1986, pp. 3-25. doi:10.1007/BF01036523

[20] Ergun, S., "Fluid Flow Through Packed Column," Chemical Engineering Progress, Vol. 48, No. 2, 1952, pp. 89-94.

[21] Innocentini, M. D., Salvini, V. R., Macedo, A., and Pandolfelli, V. C., "Prediction of Ceramic Foams Permeability Using Ergun's Equation," Materials Research, Vol. 2, No. 4, Oct. 1999. doi:10.1590/S1516-14391999000400008

[22] Philipse, A. P., and Schram, H. L., "Non-Darcian Airow Through Ceramic Foams," Journal of the American Ceramic Society, Vol. 74, No. 4, 1991, pp. 728-732. doi:10.1111/j.1151-2916.1991.tb06916.x 
[23] Straatman, A. G., Gallego, N. C., Yu, Q., and Thompson, B. E., "Characterization of Porous Carbon Foam as a Material for Compact Recuperators," Journal of Engineering for Gas Turbines and Power, Vol. 129, No. 2, April 2007, pp. 326-330. doi: $10.1115 / 1.2436562$

[24] Ward, J. C., "Turbulent Flow in Porous Media," Journal of the Hydraulics Division, American Society of Civil Engineers, Vol. 90, No. HY-5, Sept. 1964, pp. 1-12.

[25] Marschall, J., and Milos, F. S., "Gas Permeability of Rigid Fibrous Refractory Insulations," Journal of Thermophysics and Heat Transfer, Vol. 12, No. 4, Oct.-Dec. 1998, p. 8. doi: $10.2514 / 2.6372$.

[26] Marschall, J., and Cox, M. E., "Gas Permeability of Lightweight Ceramic Ablators," Journal of Thermophysics and Heat Transfer,
Vol. 13, No. 3, 1999, pp. 382-384. doi: $10.2514 / 2.6451$

[27] Suzuki, T., Sawada, K., Yamada, T., and Inatani, Y., "Experimental and Numerical Study of Pyrolysis Gas Pressure in Ablating Test Piece," Journal of Thermophysics and Heat Transfer, Vol. 19, No. 3, JulySept. 2005, pp. 266-272. doi: $10.2514 / 1.12211$

[28] Yamada, T., Sawada, K., Yamada, T., and Inatani, Y., "Thermal Response of Ablative Test Piece in Arc-Heated Wind Tunnel," AIAA Paper 2004-341, 5-8 Jan. 2004.

[29] Suzuki, T., Sawada, K., Yamada, T., and Inatani, Y., "Gas Permeability of Oblique-Layered Carbon-Cloth Ablator," Journal of Thermophysics and Heat Transfer, Vol. 18, No. 4, 2004, pp. 548-550. doi: $10.2514 / 1.6242$ 